\title{
ERRATA
}

\section{Numerical simulations of mid-ocean ridge hydrothermal circulation including the phase separation of seawater}

Yoshifumi Kawada, Shigeo Yoshida, and Sei-ichiro Watanabe

Earth Planets Space, Vol. 56, pp. 193-215, 2004

Figures 5 and 6 on p. 205 should read as follows:

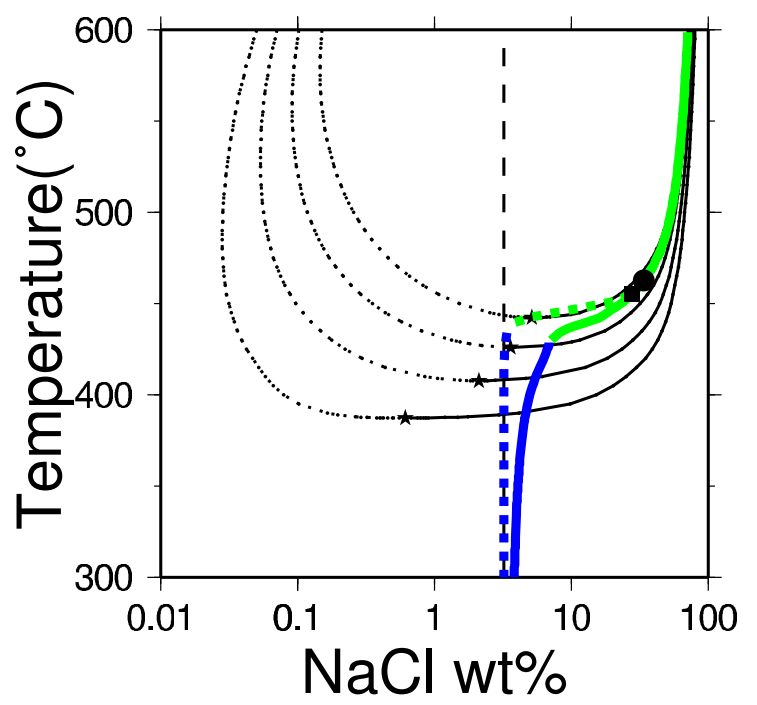

Fig. 5. Temperature and $\mathrm{NaCl}$ concentration along the left downwelling boundary (thick dotted curve) and the right upwelling boundary (thick solid curve) of the calculation area. The vertical axis is the temperature in Celsius degree, and the horizontal axis is the logarithm of NaCl concentration in wt\%. The blue curves signify one-phase fluid, and the green curves signify two-phase fluid. The filled circle and square denote $(C, T)$ at the boundaries between the convection and the stagnant layers. The two-phase co-existing curves for depths of 2500, 3000, 3500 and $4000 \mathrm{~m}$ (corresponding to pressures of 25, 30,35 and $40 \mathrm{MPa}$, respectively), which are the same as in Fig. 1, are shown.

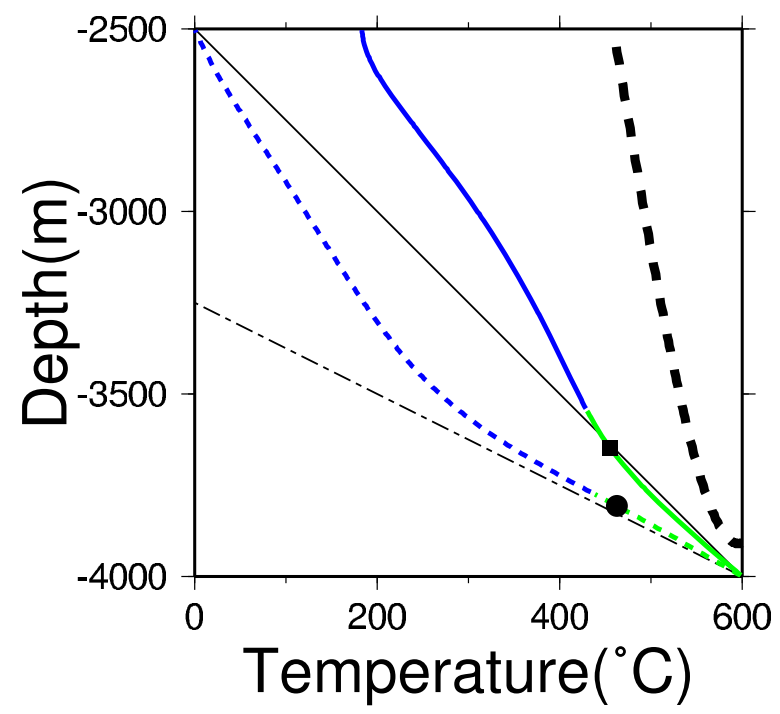

Fig. 6. Temperature as a function of depth along the left downwelling boundary (thick dotted curve) and the right upwelling boundary (thick solid curve) of the calculation area. The parameters for the calculation are $R a=100, \Pi=10$, and $1 / L e=1$. The vertical axis is the depth from the sea surface in meter, and the horizontal axis is the temperature in Celsius degree. The thin solid line shows the conductive temperature profile, and the thin dash-dotted line shows the conductive profile with the thermal conductivity being half. The thick dotted curve represents the vapor-halite-liquid triple point (Palliser and McKibbin, 1998a). Halite precipitates if the temperature of the fluid is higher than this curve. The meaning of the other symbols, colors and patterns of the curves are the same as those in Fig. 5. 
Figure 11 on p. 210 and Figure 12 on p. 211 should read as follows:
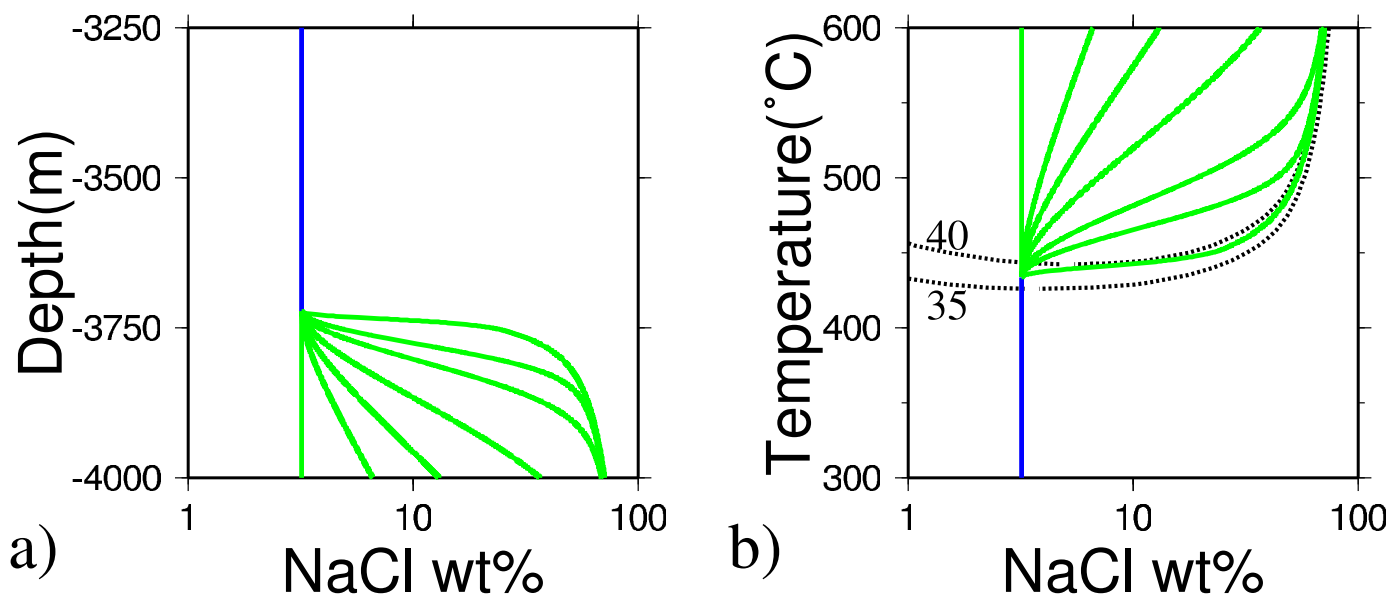

Fig. 11. Results of one-dimensional calculations for $B=0,5,10,20,50,100$, and 1000, from left to right in the figure. The non-dimensionalized temperature gradient $F$ for the calculation is 1.5 . a) depth from the seafloor against $\mathrm{NaCl}$ concentration in wt $\%$. The latter is in a logarithmic scale. b) temperature in Celsius degree against $\mathrm{NaCl}$ concentration in wt\%. The latter is in a logarithmic scale. The meaning of the other symbols, patterns and colors of the curves are the same as those in Fig. 5. Note that $B=0$ corresponds to one-phase thermal conduction.
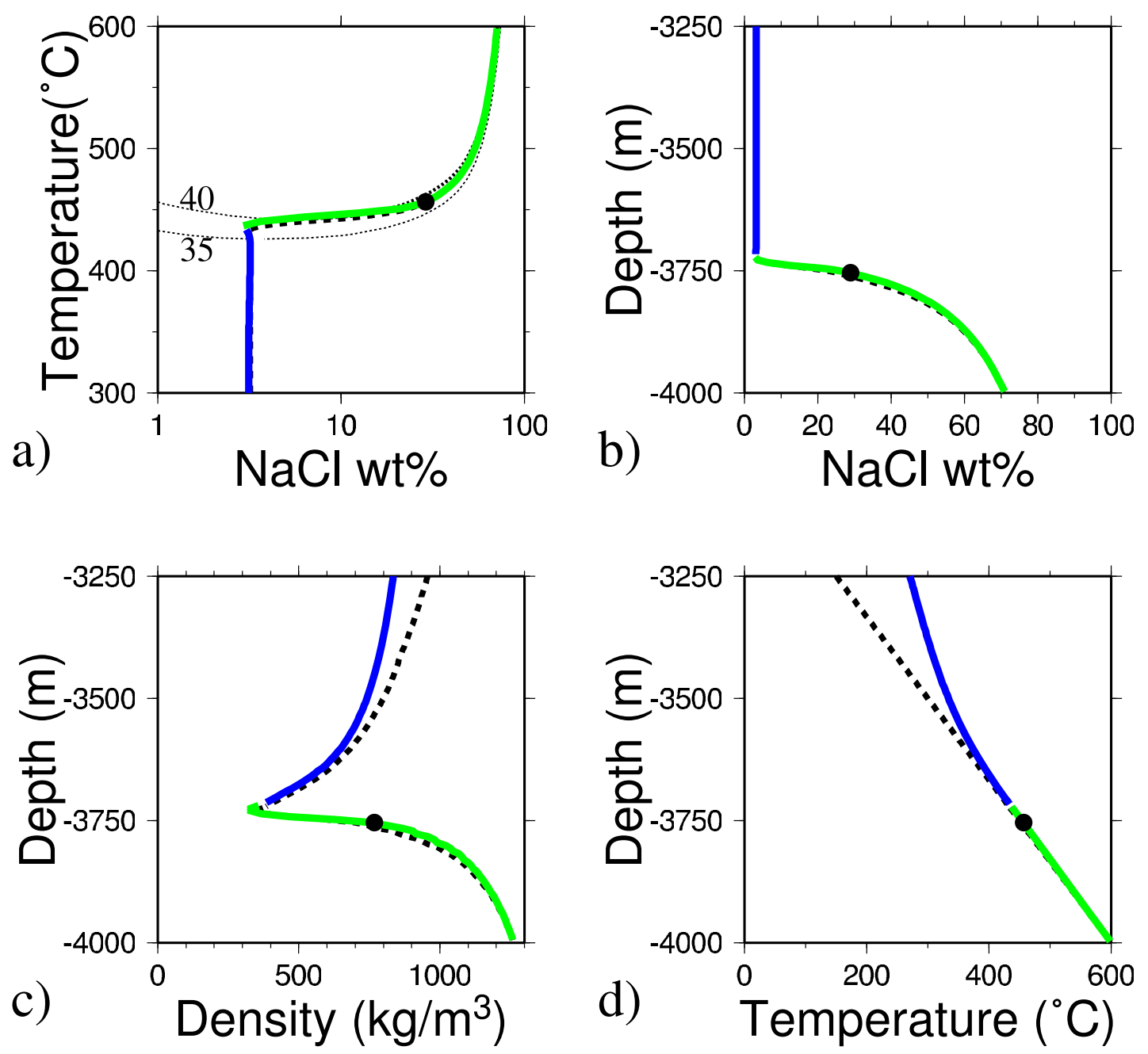

Fig. 12. Comparison of the results of one-dimensional and two dimensional calculations. a) Temperature against $\mathrm{NaCl}$ concentration, b) depth against $\mathrm{NaCl}$ concentration, $\mathrm{c}$ ) depth against density, and d) depth against temperature. The parameters used in the calculations are $R a=100, \Pi=10$, and $1 / L e=1(B=1000)$. The thin black dotted curves in a) signify the two-phase co-existing curves, which are the same as those in Fig. 5. The thick dotted curves are the result of the one-dimensional calculation for a non-dimensionalized temperature gradient $F$ of 1.5 . The colored solid curves signify the two-dimensional result along $x=0.25$ (the center of the calculation area). The one- and two-dimensional results are almost identical in the two-phase region and in the one-phase region just above the two-phase region. The meanings of the other colors and symbols are the same as those in Fig. 5. 